\title{
Automatic Appropriate Segment Extraction from Shots Based on Learning from Example Videos
}

\author{
Yousuke Kurihara, Naoko Nitta, and Noboru Babaguchi \\ Graduate School of Engineering, Osaka University \\ 2-1 Yamada-oka, Suita, Osaka, 565-0871 Japan \\ \{kurihara, naoko, babaguchi\}@nanase.comm.eng.osaka-u.ac.jp
}

\begin{abstract}
Videos are composed of shots, each of which is recorded continuously by a camera, and video editing can be considered as a process of re-sequencing shots selected from original videos. Shots usually include redundant intervals, which are often edited out by professional editors. Defining the intact interval which is used in the edited video as the appropriate segment and all other intervals of equal length as inappropriate segments, this paper proposes a method for automatically extracting appropriate segments from shots. Since what kinds of characteristics make an interval appropriate to be used in the edited video should be different among shots with different content, the proposed method firstly categorizes shots according to their content with Support Vector Machines. Then, the appropriate segments are extracted based on the temporal patterns of audio and visual features in appropriate and inappropriate segments learned with Hidden Markov Models for each shot category. The effectiveness of the proposed method is verified with experiments.
\end{abstract}

Keywords: video editing, segment extraction, shot categorization, example videos.

\section{Introduction}

Video editing is to create a new video content by combining segments selected from original videos. There are two purposes of video editing, 1)efficiently conveying information to viewers and 2)stimulating viewers' interests. As an example of automatic video editing techniques, video summarization has been realized by extracting only semantically important video shots based on metadata to achieve the former purpose [1, and by extracting important shots based on video tempo created by shot length, motion intensity, and audio energy to achieve the latter purpose 2. Although the videos created by their methods have been verified to include similar content as manually edited videos, they are still inferior in quality to professionally edited videos in terms of stimulating viewers' interests. This is attributed to the fact that most existing methods consider shots, each of which is recorded continuously by a camera, as the minimum units of video segments. However, a shot often includes redundant intervals. Therefore,

T. Wada, F. Huang, and S. Lin (Eds.): PSIVT 2009, LNCS 5414, pp. 1082 1093, 2009.

(C) Springer-Verlag Berlin Heidelberg 2009 
extracting only the essential intervals from shots is necessary to create videos which more effectively stimulate viewer's interests. In what follows, the interval of a shot which is used in the edited video is referred as an appropriate segment and all other intervals of equal length as inappropriate segments.

There are some related work for extracting appropriate segments from shots. For example, a shot was shortened so that the motion intensity, direction, and speed are similar between adjacent cut-out segments [3]. Further, important subshots [4, which were divided based on the local maximum of frame difference, speech segments [5, clips with clearly audible sound [6], and clips without excessive camera motion or overexposure [7] have been extracted as the appropriate segments. However, since the rules to evaluate the appropriateness of segments are determined manually, questions still remain in their legitimacy.

We therefore propose a method for extracting appropriate segments from shots based on temporal audio-visual patterns automatically obtained from example videos. However, since the temporal audio-visual patterns can be different among shots with different content, shots firstly need to be categorized based on their content. Therefore, the proposed method starts with learning the audio and visual characteristics of each shot category with Support Vector Machines(SVMs). Then, for each shot category, given sets of appropriate and inappropriate segments as examples, the proposed method learns the temporal patterns of how frame-level audio and visual features change in each type of segment with Hidden Markov Models(HMMs). Finally, when an original shot is given, the method determines its shot category with the learned SVMs and extracts an appropriate segment from the shot based on its likelihood value to be observed in the learned HMMs.

\section{Appropriate Segment Extraction}

Fig. 11 shows the outline of the proposed method. The proposed method consists of two phases: Learning Phase and Segment Extraction Phase. We assume that audio and visual features change in a characteristic way in appropriate segments selected by professional editors. However, since the temporal patterns in appropriate segments can be different among shots with different content, shots need to be categorized first. Therefore, in Learning Phase, after extracting a set of audio and visual features from each shot, the characteristics of each shot category are learned with SVMs. Then, given sets of appropriate and inappropriate segments as examples for each shot category, the temporal patterns of how audio and visual features change in each type of segment are learned with HMMs. Each feature is discretized to represent frames with similar audio and visual features with a symbol. Then, symbol sequence patterns in appropriate segments and inappropriate segments are learned with HMMs, generating a HMM for appropriate segments and a HMM for inappropriate segments respectively.

In Segment Extraction Phase, when an original shot and the length of the segment to be extracted are given, the shot category is determined based on the learned SVMs. A symbol sequence of the original shot is obtained and the 


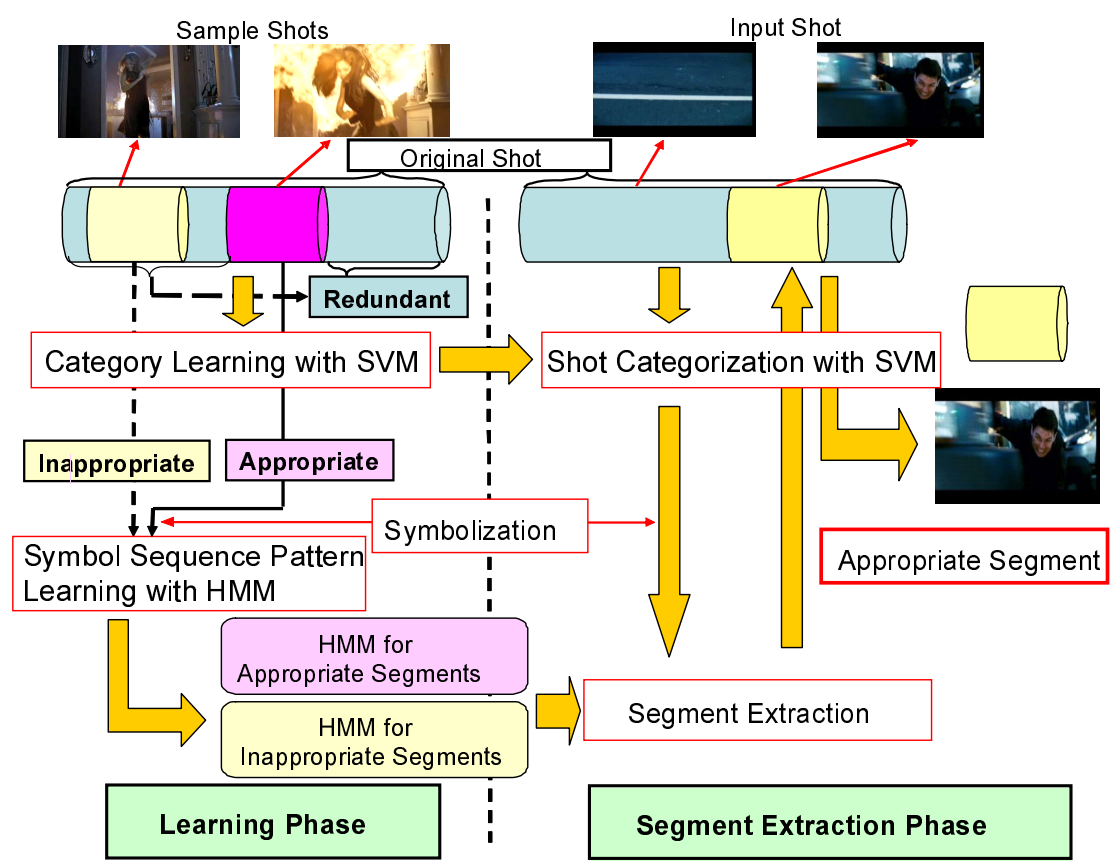

Fig. 1. Outline of the method

likelihoods for all segments of the specified length to be observed in each HMM are calculated. The segment with the maximum ratio between the likelihood values for the HMMs for appropriate segments and for inappropriate segments is extracted as the most appropriate segment. The proposed method requires the following four procedures.

1. Shot Categorization with SVMs

2. Symbolization

3. Symbol Sequence Pattern Learning with HMMs

4. Segment Extraction

\subsection{Shot Categorization with SVMs}

Audio and visual temporal patterns in appropriate segments should be different among shots with different content. For example, in shots which include camera motion or fast moving objects, large visual changes tend to attract human attention. In shots which include dialogue, human-voice intervals are considered important. This paper especially focuses on these two types of shots and defines the former shots as Action, the latter as Conversation, and the other shots as Others. 
In Learning Phase, after extracting audio or visual features which can describe the difference among three shot categories from sample shots, the proposed method firstly learns the audio-visual characteristics of each shot category.

The characteristic of each shot category is shown in Table 1. There can be large visual changes only in Action shots. Moreover, Conversation and Others differ in whether they include voice and human faces or not. We therefore use a hierarchical structure as shown in Fig. 2 to categorize shots. Shots are initially categorized into Action and Non-Action shots based on visual features. In the next step, Non-Action shots are categorized into Conversation and Others based on audio and visual features.

Since Action shots include large luminance change and their shot length is relatively short, we use the shot length, visual disturbance and average luminance difference as the visual features. Visual disturbance is computed based on the structural tensors of frames in each shot 8 . In addition, since the frequency spectrum are different between Conversation and Others shots and Zero Crossing Ratio(ZCR) varies widely in human-voice intervals, we use the mean and variance of ZCR, and Energy Ratio of SubBand at frequency band(ERSB[1/2/3]) as the audio features 9 . Furthermore, since Conversation shots usually include human faces, we compute the ratio of human face areas to the image size using the face detection algorithm in OpenCV library 10, 11.

We use SVMs for shot categorization. SVM is one of the machine learning methods, which is easily adaptable to two-category problems. Since the shots are categorized into two categories in each hierarchy, SVM is adaptable to shot categorization in the proposed method.

Finally, given an original shot, Segment Extraction Phase categorizes it as one of the three categories based on its extracted features with SVMs learned in Learn

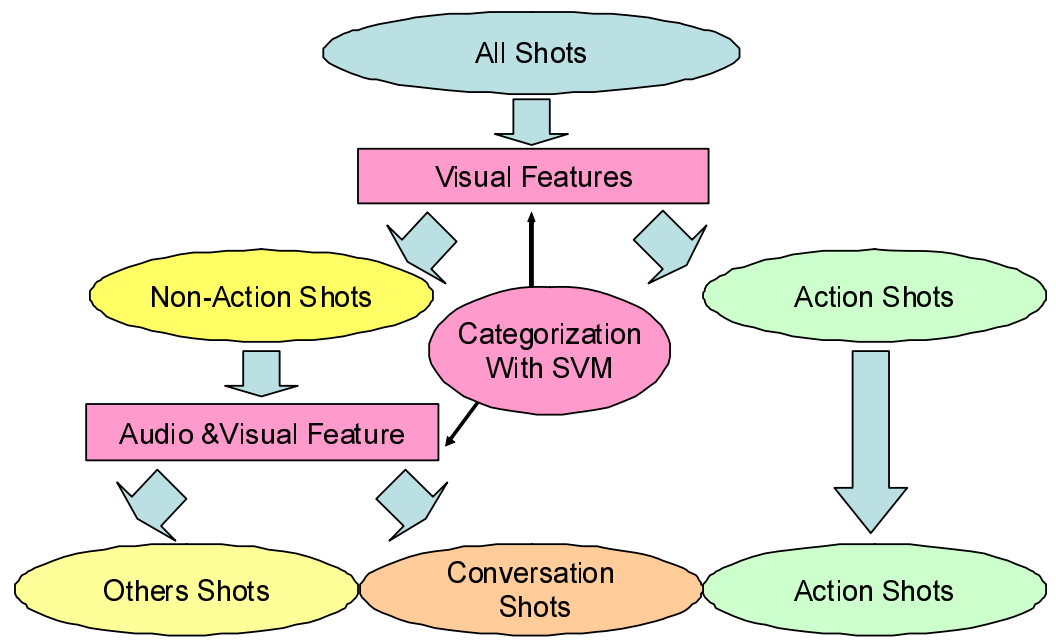

Fig. 2. Shot Categorization with SVM 
Table 1. Characteristics of Shot Category

\begin{tabular}{c|c|c}
\hline Category & Visual Characteristic & Audio Characteristic \\
\hline Action & large change & none \\
\hline Conversation & $\begin{array}{l}\text { small change } \\
\text { human faces }\end{array}$ & voice \\
\hline Others & small change & non-voice \\
\hline
\end{tabular}

\subsection{Symbolization}

After categorization, temporal patterns in the appropriate segments need to be learned with HMMs. Which audio or visual features change characteristically in appropriate segments should depend on the shot categories. Therefore, the audio and visual features which suit for each shot category need to be extracted. In this paper, focusing especially on Conversation and Action shots, we extract features suitable for each shot category 12 . Table 2 summarizes the characteristics of appropriate segments for each shot category and audio and visual features determined to be used based on the characteristics. In this table, STE stands for Short-Time Energy, ESTD for STE Standard Deviation, and LSTER for Low Short-Time Energy Ratio 9], According to the table, we extract relevant features for each category from each frame in order to see how these features change over a sequence of frames.

We use Principal Component Analysis(PCA) to reduce the noise contained in features, and as a result, to reduce the dimension of features. After discretization of each component, all features in each discretized feature vector space is represented with a symbol. Consequently, a sequence of frames is transformed into a sequence of symbols.

\subsection{Symbol Sequence Pattern Learning with HMMs}

Hidden Markov Models(HMMs) are used to learn the temporal patterns of the symbol sequence of appropriate segments and inappropriate segments. HMM is one of the popular techniques to construct an efficient model which describes the temporal patterns of data sequence 13 . The main advantages over other methods, such as Support Vector Machines and Neural Networks, are their ability to model variable-length patterns. Since the length of the appropriate segments is varied for each shot, we need to use a recognition model which can learn variable-length patterns. The symbol sequences of appropriate segments and inappropriate segments for each shot category are fed into two separate HMMs. The parameters of HMM are learned with Baum-Welch algorithm[13.

\subsection{Segment Extraction}

Finally, when an original shot and the time length of the segment to be extracted are specified by a user, the proposed method extracts an appropriate segment of the specified length based on the learned HMMs. Fig. 3 shows the flow of 
Table 2. Audio and Visual Features

\begin{tabular}{c|c|c}
\hline Shot Category & $\begin{array}{c}\text { Characteristics of } \\
\text { appropriate segments }\end{array}$ & audio and visual features \\
\hline \multirow{4}{*}{ Conversation } & $\begin{array}{c}\text { clearly audible voice } \\
\text { frequency spectrum } \\
\text { which largely differs from } \\
\text { that in non-voice segments }\end{array}$ & STE, ESTD, LSTER \\
\cline { 2 - 3 } & widely varied zero crossing ratio & ERSB \\
\hline \multirow{3}{*}{ Action } & large luminance change & Luminance \\
\cline { 2 - 3 } & widely varied zero crossing ratio & ZCR \\
\cline { 2 - 3 } & sharp loud sound effects & STE \\
\hline
\end{tabular}

segment extraction. $k$ denotes the number of frames of the appropriate segment. First, a symbol sequence is extracted from the given original shot. $h_{k}(f)$, the $\log$ likelihood for a sequence of $k$ symbols centered at the $f$ th symbol to be observed in the HMM for appropriate segments, and $g_{k}(f)$, the log likelihood for the same sequence to be observed in the HMM for inappropriate segments, are calculated with Forward algorithm 13 . Then, a segment centered at the $F$ th frame, is extracted as the most appropriate segment as follows.

$$
F=\arg \max _{f}\left(h_{k}(f)-g_{k}(f)\right)
$$

\section{Experiments}

We evaluated our proposed method using 26 action movies and their trailers. Movie trailers are one of the representative professionally edited videos, mainly designed to attract viewers' interests. Another benefit of using these videos are that both of the original videos and edited videos are easily available.

\subsection{Shot Categorization}

We categorized shots selected randomly from action movies into three categories, 1)Action, 2)Conversation, and 3)Others. The title of movies and the number of sample and test shots are shown in Table 3 and the result of shot categorization is shown in Table $4.75 .9 \%((43+39) / 108)$ of shots were correctly categorized into Action and Non-Action shots, and $71.8 \%((16+12) / 39)$ of NonAction shots were correctly categorized into Conversation and Others. However, $34.3 \%((12+14+6+5) / 108)$ of shots were incorrectly categorized.

Fig. 4 shows some examples of incorrectly categorized shots. For example, a Conversation shot (a) was incorrectly categorized as an Action due to its large visual change. Since Conversation shots tend to include close-up faces as shown in Fig. 5] small motions of characters largely increases luminance difference which makes them resemble to Action shots.

An Action shot (b) was incorrectly categorized as a Non-Action shot since the intervals of large visual change were relatively short against the shot length 


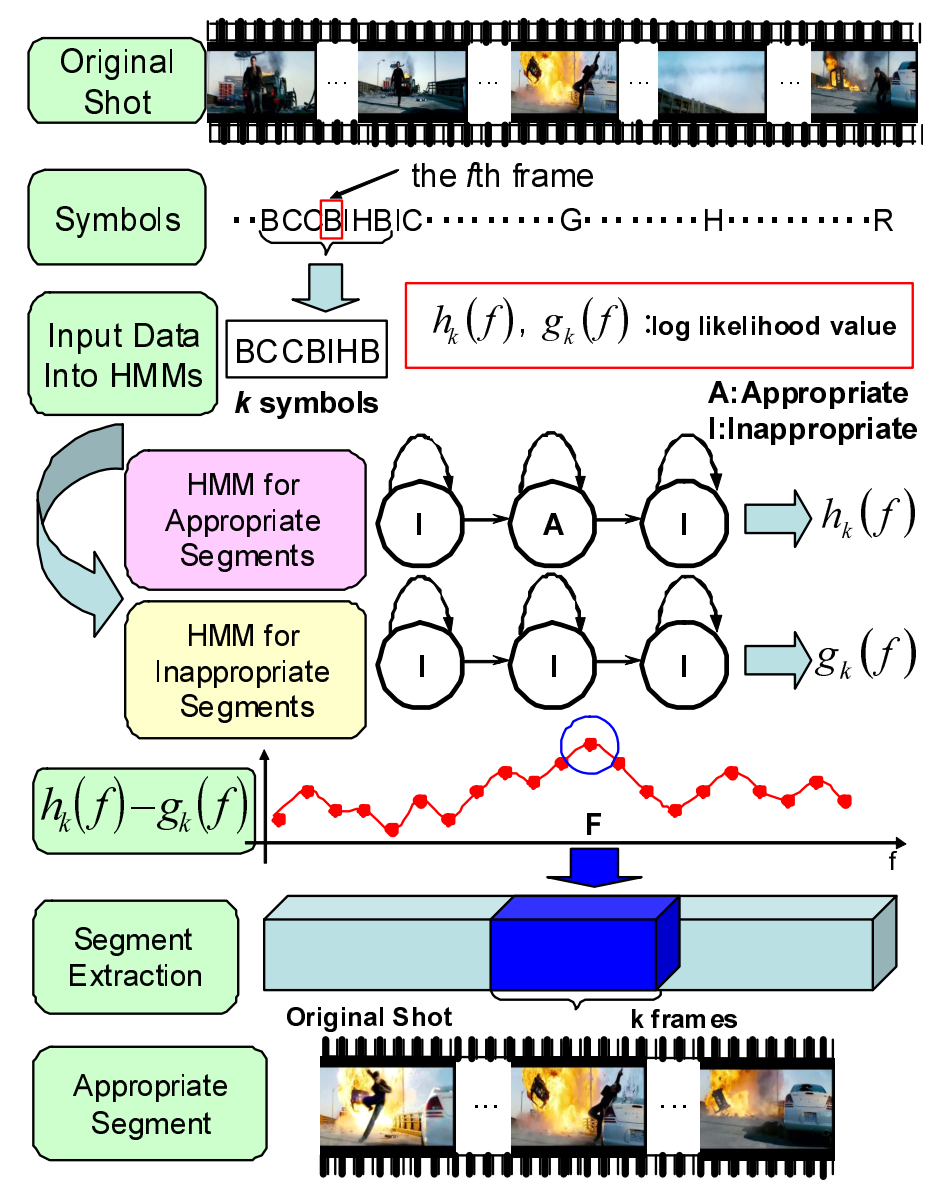

Fig. 3. Appropriate Segment Extraction

and the average luminance difference was low. Similarly, a Conversation shot (c) was incorrectly categorized as an Others shot since the length of dialogue was relatively short against the shot length and human face areas were small. In this paper, we extracted audio and visual features per frame which are influenced by the shot length as a visual feature. We therefore need to consider other features which are not influenced by the shot length, such as the max luminance difference.

Moreover, an Others shot (d) was incorrectly categorized as a Conversation since the moaning sound of the character yielded similar audio features to Conversation shots. However, since intervals which include moaning sound of the character could be essential, it could have an insignificant effect on appropriate segment extraction. 
Table 3. Sample and Test Shots for Shot Categorization

\begin{tabular}{c|c|c|c|c|c|c}
\hline \multirow{2}{*}{ Movie Title } & \multicolumn{2}{|c|}{ Action } & \multicolumn{2}{c|}{ Conversation } & \multicolumn{2}{c}{ Others } \\
\cline { 2 - 7 } & $\begin{array}{c}\text { Sample } \\
\text { Shot }\end{array}$ & $\begin{array}{c}\text { Test } \\
\text { Shot }\end{array}$ & $\begin{array}{c}\text { Sample } \\
\text { Shot }\end{array}$ & $\begin{array}{c}\text { Test } \\
\text { Shot }\end{array}$ & $\begin{array}{c}\text { Sample } \\
\text { Shot }\end{array}$ & $\begin{array}{c}\text { Test } \\
\text { Shot }\end{array}$ \\
\hline Back to the Future Part II & 9 & 0 & 10 & 0 & 7 & 0 \\
\hline Butterfly Effect & 0 & 0 & 0 & 2 & 0 & 0 \\
\hline Day After Tomorrow & 0 & 5 & 0 & 3 & 0 & 0 \\
\hline Harry Potter II & 0 & 0 & 0 & 4 & 0 & 0 \\
\hline Harry Potter III & 3 & 0 & 3 & 1 & 2 & 1 \\
\hline Harry Potter IV & 0 & 2 & 0 & 0 & 7 & 3 \\
\hline Harry Potter V & 0 & 1 & 0 & 0 & 0 & 8 \\
\hline I, ROBOT & 0 & 9 & 0 & 0 & 0 & 0 \\
\hline Live Free or Die Hard & 0 & 1 & 0 & 0 & 0 & 1 \\
\hline MI:2 & 0 & 0 & 0 & 1 & 0 & 0 \\
\hline MI:3 & 0 & 6 & 0 & 0 & 0 & 0 \\
\hline Minority Report & 5 & 0 & 6 & 4 & 0 & 0 \\
\hline Mr. and Mrs. Smith & 0 & 4 & 0 & 4 & 0 & 0 \\
\hline New Police Story & 0 & 1 & 0 & 1 & 0 & 0 \\
\hline Pirates of the Caribbean II & 0 & 7 & 0 & 3 & 0 & 0 \\
\hline Star Wars I & 0 & 0 & 0 & 2 & 0 & 0 \\
\hline Resident Evil: Apocalypse & 0 & 1 & 0 & 0 & 0 & 0 \\
\hline Star Wars II & 0 & 13 & 0 & 2 & 0 & 0 \\
\hline Star Wars III & 2 & 0 & 5 & 0 & 4 & 0 \\
\hline The Lord of the Rings I & 8 & 0 & 2 & 0 & 2 & 3 \\
\hline The Lord of the Rings II & 3 & 0 & 1 & 0 & 1 & 0 \\
\hline The Lord of the Rings III & 0 & 3 & 0 & 3 & 0 & 4 \\
\hline Transformers & 0 & 4 & 0 & 1 & 1 & 0 \\
\hline Van Helsing & 3 & 0 & 8 & 0 & 2 & 0 \\
\hline Total & 33 & 57 & 35 & 31 & 26 & 20 \\
\hline & & & & & & \\
\hline & & & & & 0 \\
\hline
\end{tabular}

\section{$3.2 \quad$ Appropriate Segment Extraction}

We extracted shots from movie trailers as the appropriate segments, and the corresponding shots as the original shot. Then, the intervals excluding the appropriate segments are extracted from the original shot as the redundant intervals. Table 5 shows the titles and the number of sample and test original shots. Here, appropriate and inappropriate segments obtained from sample original shots are used as examples and an appropriate segment is extracted from the test original shot by the proposed method.

Objective Evaluation. As an objective evaluation, we compared the appropriate segments extracted by the proposed method with the segments used in movie trailers 12, In what follows, we call segments extracted by the proposed method extracted segments, and segments used in movie trailers correct segments. In evaluation, we allowed the frame difference between the extracted segment and the correct segment up to $t$ frames. Table 6 shows the results of objective 
Table 4. Result of Shot Categorization with SVMs

\begin{tabular}{c|c|c|c}
\hline & & \multicolumn{2}{|c}{ Result of Categorization } \\
\hline & & Action & Non-Action \\
\hline \multirow{3}{*}{$\begin{array}{c}\text { Correct } \\
\text { Shot }\end{array}$} & Action & 43 & 14 \\
\cline { 2 - 4 } Category & Non-Action & 12 & 39 \\
\cline { 2 - 4 } & & Conversation & Others \\
\cline { 2 - 4 } & Conversation & 16 & 5 \\
\cline { 2 - 4 } & Others & 6 & 12 \\
\hline
\end{tabular}

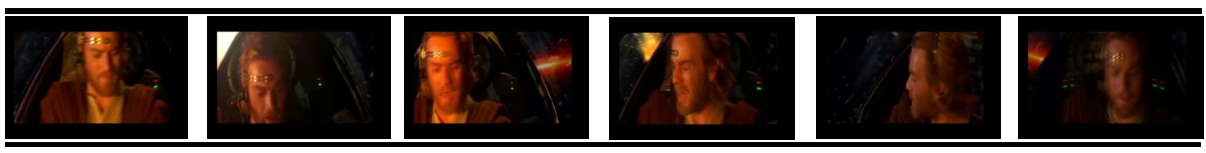

(a) Conversation $\rightarrow$ Action

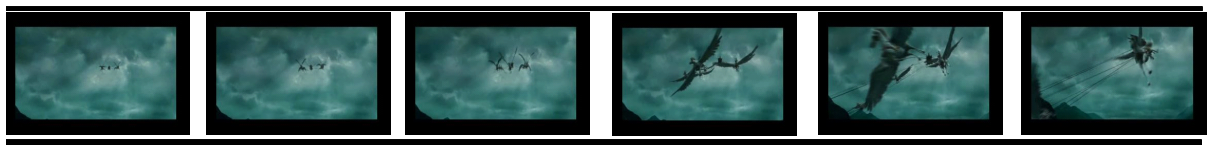

(b) Action $\rightarrow$ Non-Action

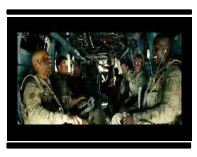

(c) Conversation $\rightarrow$ Others

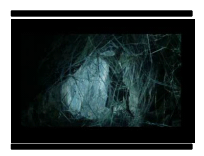

(d) Others $\rightarrow$ Conversation

Fig. 4. Examples of Incorrectly Categorized Shots

evaluation. When $t=5$, the appropriate segments were correctly extracted from $72.5 \%$ of the original shots.

Fig. 6] shows an example of extracting an appropriate segment. The original shot includes a crash scene in which a truck nearly hits a character, which corresponds to the correct segment, and the proposed method was able to extract the same segment within 3-frame difference by the proposed method.

Subjective Evaluation. As a subjective evaluation, we also conducted a questionnaire to evaluate if users feel the extracted segments are appropriate to be used in edited videos. This questionnaire was administered to 14 people. First, we presented them the original shot and then three segments as the appropriate

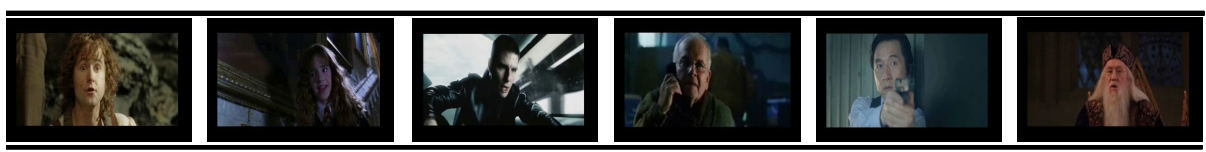

Fig. 5. Conversation Shots 
Table 5. Sample and Test Shots for Appropriate Segment Extraction

\begin{tabular}{c|c|c|c|c}
\hline \multirow{2}{*}{ Movie Title } & \multicolumn{2}{|c|}{ Action } & \multicolumn{2}{c}{ Conversation } \\
\cline { 2 - 5 } & $\begin{array}{c}\text { Sample } \\
\text { Shot }\end{array}$ & $\begin{array}{c}\text { Test } \\
\text { Shot }\end{array}$ & $\begin{array}{c}\text { Sample } \\
\text { Shot }\end{array}$ & $\begin{array}{c}\text { Test } \\
\text { Shot }\end{array}$ \\
\hline New Police Story & 1 & 0 & 1 & 1 \\
\hline MI:2 & 0 & 0 & 1 & 0 \\
\hline MI:3 & 2 & 4 & 0 & 0 \\
\hline Mr. and Mrs. Smith & 3 & 2 & 4 & 2 \\
\hline Day After Tomorrow & 1 & 3 & 2 & 1 \\
\hline Minority Report & 0 & 0 & 3 & 1 \\
\hline Butterfly Effect & 0 & 0 & 1 & 2 \\
\hline I, ROBOT & 3 & 6 & 0 & 0 \\
\hline Dead Man's Chest & 0 & 7 & 0 & 3 \\
\hline Star Wars I & 0 & 1 & 0 & 3 \\
\hline Star Wars II & 0 & 13 & 0 & 2 \\
\hline Star Wars III & 0 & 4 & 0 & 2 \\
\hline Resident Evil: Apocalypse & 0 & 1 & 0 & 0 \\
\hline Harry Potter I & 0 & 0 & 0 & 1 \\
\hline Harry Potter II & 0 & 5 & 0 & 2 \\
\hline Harry Potter III & 0 & 1 & 0 & 2 \\
\hline Total & 10 & 47 & 12 & 22 \\
\hline & & & &
\end{tabular}
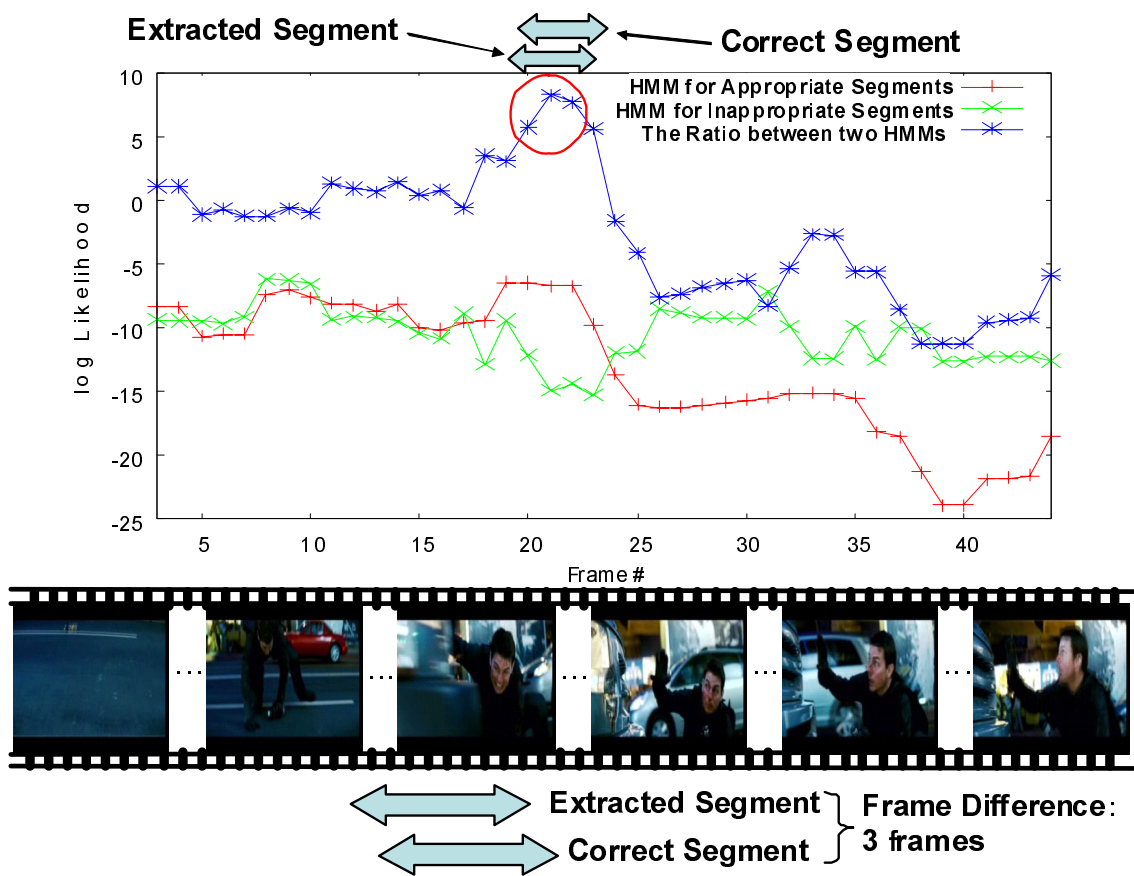

Fig. 6. An Example of Extracting an Appropriate Segment 
Table 6. Results of Objective Evaluation

\begin{tabular}{c|c|c}
\hline \multirow{2}{*}{} & \multicolumn{2}{|c}{ Accuracy } \\
\cline { 2 - 3 } & Action & Conversation \\
\hline $\mathrm{t}=2$ & $53 \%(25 / 47)$ & $60 \%(11 / 22)$ \\
\hline $\mathrm{t}=3$ & $60 \%(28 / 47)$ & $64 \%(14 / 22)$ \\
\hline $\mathrm{t}=5$ & $72 \%(34 / 47)$ & $73 \%(16 / 22)$ \\
\hline
\end{tabular}

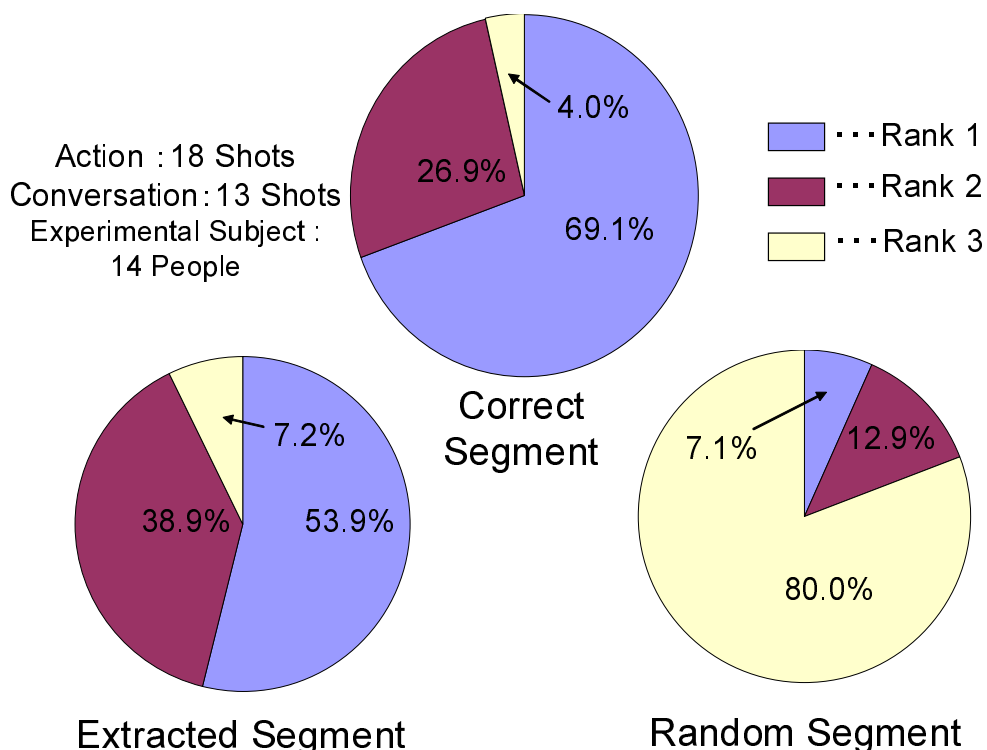

Fig. 7. Result of Subjective Evaluation

segment, 1)correct segment, 2)extracted segment, and 3)random segment, and asked them to rank the three segments in terms of their adequacy 12 . In this questionnaire, we allowed them to rank more than two segments in the same rank. Fig. 7 shows the results of the subjective evaluation. These results have verified that the proposed method was able to extract subjectively appropriate segments.

\section{Conclusion}

In this paper, we proposed a method for automatically extracting an appropriate segment from an original shot based on the temporal patterns of how the audio and visual features change both in the appropriate and inappropriate segments, which are automatically learned from examples. The proposed method is composed of shot categorization with SVMs and segment extraction with HMMs. 
We experimented the proposed method using examples obtained from movies and their trailers. According to the experiments, $75.4 \%$ of shots were correctly categorized into Action and Non-Action shots, and $71.8 \%$ of Non-Action shots were correctly categorized into Conversation and Others. However there is still need for more argument about which audio and visual features should be used to categorize shots. Results of objective and subjective evaluation have verified the effectiveness of the proposed method in extracting appropriate segments. We need to consider how to automatically determine the number of frames of appropriate segments to be extracted.

\section{References}

1. Takahashi, Y., Nitta, N., Babaguchi, N.: Video Summarization for Large Sports Video Archives. In: Proceedings of ICME 2005 (July 2005)

2. Chen, H.W., Kuo, J.H., Chu, W.T., Wu, J.L.: Action Movies Segmentation and Summarization Based on Tempo Analysis. In: Proceedings of MIR 2004, pp. 251258 (October 2004)

3. Takemoto, R., Yoshitaka, A., Hirashima, T.: Hirashima: Video Editing based on Movie Effects by Shot Length Transition, Technical Report of IEICE PRMU 2005149-183, pp.19-24 (January 2006)

4. Hua, X.S., Lu, L., Zhang, H.J.: Optimization-Based Automated Home Video Editing System. IEEE Transactions on TCSVT 2004 14(5) (May 2004)

5. Aoyanagi, S., Kourai, K., Sato, K., Takada, T., Sgawara, T.: Evaluation of New Video Skimming Method Using Audio and Video Information. In: Proceedings of DEWS 2003 2-A-01 (March 2003)

6. Lienhart, R.: Abstracting Home Video Automatically. In: Proceedings of ACMMM 1999, pp. 37-40 (1999)

7. Foote, J., Cooper, M., Girgenshon, A.: Creating Music Videos using Automatic Media Analysis. In: Proceedings of ACMMM 2002, pp. 553-560 (December 2002)

8. Rasheed, Z., Shah, M.: Video Categorization Using Semantics and Semiotics. In: VIDEO MINING, pp. 185-217. Kluwer Academic Publishers, Dordrecht (2003)

9. Wang, Y., Liu, Z., Huang, J.C.: Multimedia Content Analysis Using Both Audio and Visual Clues. IEEE Signal Processing Magagine, 12-36 (November 2000)

10. http://www.intel.com/research/mrl/research/opencv/ OpenCV library, Intel

11. Viola, P., Jones, M.: Rapid object detection using a boosted cascade of simple features. In: Proceedings of CVPR 2001, pp. 511-518 (December 2001)

12. Kurihara, Y., Nitta, N., Babaguchi, N.: Appropriate segment extraction from shots based on temporal patterns of example videos. In: Satoh, S., Nack, F., Etoh, M. (eds.) MMM 2008. LNCS, vol. 4903, pp. 253-264. Springer, Heidelberg (2008)

13. Rabiner, L.R.: A Tutorial on Hidden Markov Models and Selected Applications in Speech Recognition. Proceeding IEEE 77, 257-285 (1989) 